\title{
Optimal harvesting policy of a stochastic delay predator-prey model with Lévy jumps
}

\author{
Meiling Deng \\ School of Mathematical Science, Huaiyin Normal University, Huaian 223300, P. R. China.
}

Communicated by D. Baleanu

\begin{abstract}
This paper considers the optimal harvesting of a stochastic delay predator-prey model with Lévy jumps. The traditional optimal harvesting problem of this type of model is difficult because it is difficult to get the explicit solutions of the model or to solve the corresponding delay Fokker-Planck equation of the model. In this paper, we use an ergodic method to study this problem, and establish the sufficient and necessary conditions for the existence of an optimal harvesting strategy of the model. In addition, we gain the explicit forms of the optimal harvesting effort and the maximum of the cost function. One can see that the ergodic method used in this paper can avoid solving both the model and the corresponding delay Fokker-Planck equation. (C)2017 All rights reserved.
\end{abstract}

Keywords: Predator-prey system, random perturbations, delay, optimal harvesting. 2010 MSC: 92D25, 60H10, 60H30.

\section{Introduction}

Optimal harvesting problem is one of the most important and interesting topics in mathematical biology. Under the classical catch-per-unit-effort (CPUE) harvesting hypothesis, Clark [6] established the optimal harvesting strategy for a deterministic logistic model. From then on, optimal harvesting problems have attract more and more attention. Many deterministic population models with harvesting have been proposed and studied. However, population models are inevitably affected by various stochastic perturbations. Thus in recent years, stochastic population models with harvesting have received great attention [5, 11, 32]. For example, Beddington and May [5] considered the optimal harvesting problem of a stochastic logistic model by solving the corresponding Fokker-Planck equation of the model. Then the results in [5] was extended to a general stochastic logistic model [11]. Zou et al. [32] investigated the optimal harvesting problem of a stochastic Gompertz model by using the explicit solution of the model.

On the other hand, the natural world is full of time delay. Several authors have pointed out that time delay should not be neglected (see, e.g. [21-25]). In addition, the models in [5, 11, 32] only considered the white noises. However, population models in the natural world are inevitably affected by severe and sudden environmental perturbations, such as earthquakes, epidemics, and so on. For example, Tangshan earthquake in China, Three Mile Island nuclear accident in USA, Fukushima Daiichi nuclear disaster in 
Japan, and others. These sudden environmental perturbations cause jumps in population models, which can not be described by white noises. Several authors have suggested that one may use Lévy jumps to describe these phenomena [2,16, 33,34]. Moreover, predator-prey is a common phenomenon in the natural world [26]. Therefore, it is important to study the optimal harvesting problem of stochastic delay predator-prey model with Lévy jumps and harvesting. To the best of our knowledge, no results of this aspect have been reported. Motivated by these, in this paper, we consider the following stochastic delay predator-prey model with Lévy jumps and harvesting:

$$
\left\{\begin{aligned}
d y_{1}(t)= & y_{1}\left(t^{-}\right)\left[r_{1}-h_{1}-c_{11} y_{1}\left(t^{-}\right)-c_{12} y_{2}\left(t^{-}-\tau_{1}\right)\right] d t+\sigma_{1} y_{1}\left(t^{-}\right) d B_{1}(t) \\
& +\int_{\mathbb{Y}} y_{1}\left(t^{-}\right) \gamma_{1}(u) \widetilde{N}(d t, d u) \\
d y_{2}(t)= & y_{2}\left(t^{-}\right)\left[-r_{2}-h_{2}+c_{21} y_{1}\left(t^{-}-\tau_{2}\right)-c_{22} y_{2}\left(t^{-}\right)\right] d t+\sigma_{2} y_{2}\left(t^{-}\right) d B_{2}(t) \\
& +\int_{\mathbb{Y}} y_{2}\left(t^{-}\right) \gamma_{2}(u) \widetilde{N}(d t, d u)
\end{aligned}\right.
$$

with initial data

$$
y(\theta)=\left(y_{1}(\theta), y_{2}(\theta)\right)^{\top}=\left(\varphi_{1}(\theta), \varphi_{2}(\theta)\right)^{\top} \in \Upsilon,
$$

where $y_{1}(t)$ is the size of the prey population at time $t ; y_{2}(t)$ is the size of the predator population at time $t ; y_{i}\left(t^{-}\right)$denotes the left limit of $y_{i}(t), i=1,2 ; r_{1}>0$ stands for the intrinsic growth rate of the prey population; $r_{2}>0$ stands for the death rate of the predator population; $h_{i} \geqslant 0$ is the harvesting effort of the $i$ th species, $i=1,2 ; c_{i i}>0$ denotes the intra-specific competition coefficient of the species $i ; c_{12}>0$ is the capture rate; $c_{21}>0$ represents the efficiency of food conversion; $\sigma_{i}^{2}$ represents the intensity of the white noise, $\tau_{i} \geqslant 0$ represents the delay, $i=1,2 ; B(t)=\left(B_{1}(t), B_{2}(t)\right)^{\top}$ is a standard Brownian motion defined on a complete probability space $\left(\Omega, \mathcal{F},\left\{\mathcal{F}_{t}\right\}_{t \geqslant 0}, \mathbb{P}\right) ; \tau=\max \left\{\tau_{1}, \tau_{2}\right\}, \curlyvee$ is the family of all bounded and continuous functions from $[-\tau, 0]$ to $\mathbb{R}_{+}^{2}=\left\{y=\left(y_{1}, y_{2}\right)^{\top} \in \mathbb{R}^{2} \mid y_{i}>0, i=1,2\right\}$; $\widetilde{N}(d t, d u)=N(d t, d u)-\lambda(d u) d t$; and $N$ is a Poisson counting measure with characteristic measure $\lambda$ on a measurable subset $\mathbb{Y}$ of $(0, \infty)$ with $\lambda(\mathbb{Y})<\infty$.

The main purpose of this paper is to gain the optimal harvesting effort $h^{*}=\left(h_{1}^{*}, h_{2}^{*}\right)^{\top}$ such that (a) expectation of sustainable yield (ESY)

$$
Y(h)=\lim _{t \rightarrow \infty} \sum_{i=1}^{2} \mathbb{E}\left(h_{i} y_{i}(t)\right)
$$

is maximum;

(b) both $y_{1}$ and $y_{2}$ are persistent.

There are two traditional approaches to study the optimal harvesting problems of stochastic population model. One is to solve the corresponding Fokker-Planck equation $[5,11]$, the other is to use the explicit solution of the model [32]. However, for most stochastic delay population models, it is difficult to get the explicit solutions of the model or to solve the corresponding delay Fokker-Planck equation. In this paper, we use an ergodic method to study this problem, and establish the sufficient and necessary conditions for the existence of an optimal harvesting policy of the model. It is easy to see that the ergodic method can avoid solving both the model and the corresponding delay Fokker-Planck equation.

\section{Main results}

As a standing assumption, in this paper, we assume that $N, B_{1}$, and $B_{2}$ are independent. We also assume the following.

Assumption 2.1. There exists a constant $k>0$ such that

$$
1+\gamma_{\mathfrak{i}}(u)>0, u \in \mathbb{Y}, \int_{\mathbb{Y}} \ln ^{2}\left(1+\gamma_{\mathfrak{i}}(u)\right) \lambda(\mathrm{d} u) \leqslant k, \mathfrak{i}=1,2 .
$$

Biologically, this assumption means that the intensity of the Lévy jumps is not too large. 
For simplicity, let us define the following symbols:

$$
\begin{aligned}
\overline{g(t)} & =t^{-1} \int_{0}^{t} g(s) d s, \quad C=\left[\begin{array}{cc}
c_{11} & c_{12} \\
-c_{21} & c_{22}
\end{array}\right], \\
b_{1} & =r_{1}-h_{1}-0.5 \sigma_{1}^{2}-\int_{\Im}\left(\gamma_{1}(u)-\ln \left(1+\gamma_{1}(u)\right)\right) \lambda(d u), \\
b_{2} & =r_{2}+h_{2}+0.5 \sigma_{2}^{2}+\int_{\mathbb{Y}}\left(\gamma_{2}(u)-\ln \left(1+\gamma_{2}(u)\right)\right) \lambda(d u), \\
\Lambda & =c_{11} c_{22}+c_{12} c_{21}, \Lambda_{1}=b_{1} c_{22}+b_{2} c_{12}, \Lambda_{2}=b_{1} c_{21}-b_{2} c_{11} .
\end{aligned}
$$

Firstly, let us present several lemmas.

Lemma 2.2 ([13]). Under Assumption 2.1, for any initial value $\varphi(\theta) \in \Upsilon$, model (1.1) has a unique global solution $\mathrm{y}(\mathrm{t})=\left(\mathrm{y}_{1}(\mathrm{t}), \mathrm{y}_{2}(\mathrm{t})\right)^{\top}$ on $\mathrm{t} \geqslant-\tau$ and the solution will remain in $\mathbb{R}_{+}^{2}$ a.s. (almost surely). Moreover,

(A) if $\mathrm{b}_{1}<0$ then both the prey $\mathrm{y}_{1}$ and the predator $\mathrm{y}_{2}$ go to extinction almost surely (a.s.), i.e., $\lim _{\mathrm{t} \rightarrow \infty} \mathrm{y}_{\mathrm{i}}(\mathrm{t})=$ 0 a.s., $i=1,2$;

(B) if $\mathrm{b}_{1}>0$ and $\Lambda_{2}<0$, then the predator goes to extinction a.s. and the prey is persistent in the mean a.s., i.e.,

$$
\lim _{t \rightarrow \infty} \overline{y_{1}(t)}=\frac{b_{1}}{c_{11}}, \text { a.s.; }
$$

(C) if $\Lambda_{2}>0$, then both the prey and the predator are persistent in the mean a.s., i.e.,

$$
\lim _{\mathrm{t} \rightarrow \infty} \overline{\mathrm{y}_{1}(\mathrm{t})}=\frac{\Lambda_{1}}{\Lambda}, \quad \lim _{\mathrm{t} \rightarrow \infty} \overline{\mathrm{y}_{2}(\mathrm{t})}=\frac{\Lambda_{2}}{\Lambda}, \text { a.s. }
$$

Remark 2.3. Similar to the proof of Theorem 1 in [13], one can easily show that

(D) if $b_{1}=0$, then both the prey and the predator are non-persistent, i.e.,

$$
\lim _{t \rightarrow \infty} t^{-1} \int_{0}^{t} y_{i}(s) d s=0 \text {, a.s., } i=1,2 \text {; }
$$

(E) if $\Lambda_{2}=0$, then

$$
\lim _{t \rightarrow \infty} t^{-1} \int_{0}^{t} y_{1}(s) d s=\frac{b_{1}}{c_{11}}, \quad \lim _{t \rightarrow \infty} t^{-1} \int_{0}^{t} y_{2}(s) d s=0, \text { a.s.. }
$$

By (A)-(E), it is obvious that both the prey and the predator are persistent if and only if $\Lambda_{2}>0$.

Lemma 2.4. For all $\mathrm{p}>0$, there exists a positive constant $\mathrm{K}(\mathrm{p})$ such that $\limsup _{\mathrm{t} \rightarrow \infty} \mathbb{E} y_{i}^{\mathrm{p}}(\mathrm{t}) \leqslant \mathrm{K}(\mathrm{p}), i=1,2$.

Proof. The proof is similar to that of [10] and we omit it.

Lemma 2.5. If $\mathrm{c}_{11}>\mathrm{c}_{21}$ and $\mathrm{c}_{22}>\mathrm{c}_{12}$, then system (1.1) is stable in distribution, that is to say, there exists a unique probability measure $v(\cdot)$ such that for any $\varphi(\theta) \in \Upsilon$, the transition probability $\mathrm{p}(\mathrm{t}, \varphi, \cdot)$ of $\mathrm{y}(\mathrm{t})$ weakly converges to $v(\cdot)$ as $\mathrm{t} \rightarrow \infty$.

Proof. Let $\left(y_{1}(t ; \varphi), y_{2}(t ; \varphi)\right)^{\top}$ and $\left(y_{1}(t ; \phi), y_{2}(t ; \phi)\right)^{\top}$ be any two solutions of system (1.1) with initial data $\varphi(\theta) \in \Upsilon$ and $\phi(\theta) \in \Upsilon$, respectively. Define

$$
W(t)=\sum_{i=1}^{2}\left|\ln y_{i}(t ; \varphi)-\ln y_{i}(t ; \phi)\right|+c_{12} \int_{t-\tau_{1}}^{t}\left|y_{2}(s ; \varphi)-y_{2}(s ; \phi)\right| d s+c_{21} \int_{t-\tau_{2}}^{t}\left|y_{1}(s ; \varphi)-y_{1}(s ; \phi)\right| d s .
$$


By virtue of Itô's formula, we obtain

$$
\begin{aligned}
d W(t)= & \operatorname{sgn}\left(y_{1}(t ; \varphi)-y_{1}(t ; \phi)\right)\left[-c_{11}\left(y_{1}(t ; \varphi)-y_{1}(t ; \phi)\right)-c_{12}\left(y_{2}\left(t-\tau_{1} ; \varphi\right)-y_{2}\left(t-\tau_{1} ; \phi\right)\right)\right] d t \\
& +\operatorname{sgn}\left(y_{2}(t ; \varphi)-y_{2}(t ; \phi)\right)\left[c_{21}\left(y_{1}\left(t-\tau_{2} ; \varphi\right)-y_{1}\left(t-\tau_{2} ; \phi\right)\right)-c_{22}\left(y_{2}(t ; \varphi)-y_{2}(t ; \phi)\right)\right] d t \\
& +\sum_{i=1}^{2} \sum_{j=1, j \neq i}^{2} c_{i j}\left|y_{j}(t ; \varphi)-y_{j}(t ; \phi)\right| d t-\sum_{i=1}^{2} \sum_{j=1, j \neq i}^{2} c_{i j}\left|y_{j}\left(t-\tau_{i} ; \varphi\right)-y_{j}\left(t-\tau_{i} ; \phi\right)\right| d t \\
\leqslant & -\sum_{i=1}^{2} c_{i i}\left|y_{i}(t ; \varphi)-y_{i}(t ; \phi)\right| d t+\sum_{i=1}^{2} \sum_{j=1, j \neq i}^{2} c_{i j}\left|y_{j}\left(t-\tau_{i} ; \varphi\right)-y_{j}\left(t-\tau_{i} ; \phi\right)\right| d t \\
& +\sum_{i=1}^{2} \sum_{j=1, j \neq i}^{2} c_{i j}\left|y_{j}(t ; \varphi)-y_{j}(t ; \phi)\right| d t-\sum_{i=1}^{2} \sum_{j=1, j \neq i}^{2} c_{i j}\left|y_{j}\left(t-\tau_{i} ; \varphi\right)-y_{j}\left(t-\tau_{i} ; \phi\right)\right| d t \\
= & -\left[c_{11}-c_{21}\right]\left|y_{1}(t ; \varphi)-y_{1}(t ; \phi)\right| d t-\left[c_{22}-c_{12}\right]\left|y_{2}(t ; \varphi)-y_{2}(t ; \phi)\right| d t .
\end{aligned}
$$

Thereby,

$$
\mathbb{E}(W(t)) \leqslant W(0)-\left[c_{11}-c_{21}\right] \int_{0}^{t} \mathbb{E}\left|y_{1}(s ; \varphi)-y_{1}(s ; \phi)\right| d s-\left[c_{22}-c_{12}\right] \int_{0}^{t} \mathbb{E}\left|y_{2}(s ; \varphi)-y_{2}(s ; \phi)\right| d s .
$$

By $W(t) \geqslant 0$, one can obtain that

$$
\left[c_{11}-c_{21}\right] \int_{0}^{t} \mathbb{E}\left|y_{1}(s ; \varphi)-y_{1}(s ; \phi)\right| d s+\left[c_{22}-c_{12}\right] \int_{0}^{t} \mathbb{E}\left|y_{2}(s ; \varphi)-y_{2}(s ; \phi)\right| d s \leqslant W(0)<\infty .
$$

Hence,

$$
\mathbb{E}\left|y_{i}(t ; \varphi)-y_{i}(t ; \phi)\right| \in L^{1}[0, \infty), i=1,2 .
$$

On the other hand, by the first equation in system (1.1), we get

$$
\mathbb{E}\left(y_{1}(t)\right)=y_{1}(0)+\int_{0}^{t}\left[\left(r_{1}-h_{1}\right) \mathbb{E}\left(y_{1}(s)\right)-c_{11} \mathbb{E}\left(y_{1}^{2}(s)\right)-c_{12} \mathbb{E}\left(y_{1}(s) y_{2}\left(s-\tau_{1}\right)\right)\right] d s
$$

Thereby, $\mathbb{E}\left(y_{1}(t)\right)$ is continuously differentiable. Furthermore, in view of Lemma 2.4, we have

$$
\frac{d \mathbb{E}\left(y_{1}(t)\right)}{d t} \leqslant\left(r_{1}-h_{1}\right) \mathbb{E}\left(y_{1}(t)\right) \leqslant \widetilde{K},
$$

where $\widetilde{K}>0$ is a constant. Consequently, $\mathbb{E}\left(y_{1}(t)\right)$ is uniformly continuous. Analogously, we get that $\mathbb{E}\left(y_{2}(t)\right)$ is also uniformly continuous. It then follows from Barbalat's conclusion [3] that

$$
\lim _{t \rightarrow \infty} \mathbb{E}\left|y_{i}(t ; \varphi)-y_{i}(t ; \phi)\right|=0, i=1,2
$$

Let $\mathcal{P}(t, \varphi, A)$ be the probability of event $y(t ; \varphi) \in A$ with initial data $\varphi(\theta) \in \Upsilon$. By Lemma 2.4 and Chebyshev's inequality, we get that the family of $\{p(t, \varphi, d x)\}$ is tight $([12,14,15])$. Denote by $\mathbb{P}(\Upsilon)$ all the probability measures on $\Upsilon$. For all $P_{1}, P_{2} \in \mathbb{P}$, define

$$
d_{F}\left(P_{1}, P_{2}\right)=\sup _{f \in F}\left|\int_{\mathbb{R}_{+}^{2}} f(x) P_{1}(d x)-\int_{\mathbb{R}_{+}^{2}} f(x) P_{2}(d x)\right|,
$$


where

$$
F=\{f: \curlyvee \rightarrow \mathbb{R}|| f(x)-f(z)|\leqslant\|x-z\|,| f(\cdot) \mid \leqslant 1\} .
$$

For arbitrary $f \in F$ and $t, s>0$,

$$
\begin{aligned}
|\mathbb{E} f(y(t+s ; \varphi))-\mathbb{E f}(y(t ; \varphi))| & =\left|\mathbb{E}\left[\mathbb{E}\left(f(y(t+s ; \varphi)) \mid \mathcal{F}_{s}\right)\right]-\mathbb{E f}(y(t ; \varphi))\right| \\
& =\left|\int_{\mathbb{R}_{+}^{2}} \mathbb{E f}(y(t ; \xi)) p(s, \varphi, d \xi)-\mathbb{E f}(y(t ; \varphi))\right| \\
& \leqslant \int_{\mathbb{R}_{+}^{2}}|\mathbb{E} f(y(t ; \xi))-\mathbb{E} f(y(t ; \varphi))| p(s, \varphi, d \xi) .
\end{aligned}
$$

By (2.2), one can see that there exists a random time $T$ such that for $t \geqslant T$,

$$
\sup _{f \in F}|\mathbb{E} f(y(t ; \xi))-\mathbb{E} f(y(t ; \varphi))| \leqslant \epsilon .
$$

Namely,

$$
|\mathbb{E} f(y(t+s ; \varphi))-\mathbb{E f}(y(t ; \varphi))| \leqslant \epsilon .
$$

It then follows from the arbitrariness of $f$ that

$$
\sup _{f \in F}|\mathbb{E} f(y(t+s ; \varphi))-\mathbb{E} f(y(t ; \varphi))| \leqslant \epsilon .
$$

Hence,

$$
\mathrm{d}_{\mathrm{F}}(\mathrm{p}(\mathrm{t}+\mathrm{s}, \varphi, \cdot), \mathrm{p}(\mathrm{t}, \varphi, \cdot)) \leqslant \epsilon, \quad \forall t \geqslant T, s>0 .
$$

That is to say, $\{p(t, \rho, \cdot): t \geqslant 0\}$ is Cauchy in $\mathbb{P}$, where $\rho \equiv(0.1,0.1)^{\top}$. Then there is a unique $v(\cdot) \in \mathbb{P}(\Upsilon)$ such that

$$
\lim _{t \rightarrow+\infty} d_{F}(p(t, \rho, \cdot), v(\cdot))=0
$$

By (2.2),

$$
\lim _{t \rightarrow+\infty} d_{F}(p(t, \varphi, \cdot), p(t, \rho, \cdot))=0
$$

Hence

$$
\lim _{t \rightarrow+\infty} d_{F}(p(t, \varphi, \cdot), v(\cdot)) \leqslant \lim _{t \rightarrow+\infty} d_{F}(p(t, \varphi, \cdot), p(t, \rho, \cdot))+\lim _{t \rightarrow+\infty} d_{F}(p(t, \rho, \cdot), v(\cdot))=0,
$$

then the required assertion follows.

Our main result is the following theorem.

Theorem 2.6. For system (1.1), let Assumption 2.1, $\mathrm{c}_{11}>\mathrm{c}_{21}$, and $\mathrm{c}_{22}>\mathrm{c}_{12}$ hold. Denote

$$
\eta=\left(\eta_{1}, \eta_{2}\right)^{\top}=\left[C\left(C^{-1}\right)^{\top}+I\right]^{-1} B
$$

where

$$
\mathrm{B}=\left(\mathrm{r}_{1}-0.5 \sigma_{1}^{2}-\int_{\mathbb{Y}}\left(\gamma_{1}(\mathrm{u})-\ln \left(1+\gamma_{1}(u)\right)\right) \lambda(\mathrm{du}),-\mathrm{r}_{2}-0.5 \sigma_{2}^{2}-\int_{\mathbb{Y}}\left(\gamma_{2}(u)-\ln \left(1+\gamma_{2}(u)\right)\right) \lambda(\mathrm{du})\right)^{\top}
$$

and $\mathrm{I}$ is the unit matrix.

(i) If $\left.\Lambda_{2}\right|_{h_{i}=\eta_{i}, i=1,2}>0, C^{-1}+\left(C^{-1}\right)^{\top}$ is positive semi-definite, and $\eta_{i} \geqslant 0, i=1,2$, then the optimal harvesting effort is $\mathrm{h}^{*}=\eta=\left[\mathrm{C}\left(\mathrm{C}^{-1}\right)^{\top}+\mathrm{I}\right]^{-1} \mathrm{~B}$ and the maximum of ESY is

$$
\mathrm{Y}\left(\mathrm{h}^{*}\right)=\eta^{\top} \mathrm{C}^{-1}(\mathrm{~B}-\eta) \text {. }
$$

(ii) If the conditions in (i) do not hold, then the optimal harvesting policy does not exist. 
Proof. Let $H=\left\{h=\left(h_{1}, h_{2}\right)^{\top} \in \mathbb{R}^{2} \mid \Lambda_{2}>0, h_{i} \geqslant 0, i=1,2\right\}$. It is easy to check that for every $h \in H,(2.1)$ holds; and if the optimal harvesting effort $h^{*}$ exists, then $h^{*} \in H$.

(i) Notice that $\eta \in H$, then $H$ is not an empty set. By (2.1), one can observe that for all $h \in H$,

$$
\lim _{t \rightarrow \infty} t^{-1} \int_{0}^{t} h^{\top} y(s) d s=\sum_{i=1}^{2} h_{i} \lim _{t \rightarrow \infty} t^{-1} \int_{0}^{t} y_{i}(s) d s=h^{\top} C^{-1}(B-h) .
$$

By virtue of Lemma 2.5, one can find that system (1.1) has a unique invariant measure $v(\cdot)$. It then follows from Corollary 3.4.3 in [7] that $v(\cdot)$ is strong mixing. In view of Theorem 3.2.6 in [7], one can obtain that $v(\cdot)$ is ergodic. By (3.3.2) in [7], we have

$$
\lim _{t \rightarrow \infty} t^{-1} \int_{0}^{t} h^{\top} y(s) d s=\int_{\mathbb{R}_{+}^{2}} h^{\top} y v(d y) .
$$

Let $\vartheta(y)$ be the stationary probability density of system (1.1), then we have

$$
Y(h)=\lim _{t \rightarrow \infty} \sum_{i=1}^{2} \mathbb{E}\left(h_{i} y_{i}(t)\right)=\lim _{t \rightarrow \infty} \mathbb{E}\left(h^{\top} y(t)\right)=\int_{\mathbb{R}_{+}^{2}} h^{\top} y \vartheta(y) d y .
$$

Since the invariant measure of system (1.1) is unique, then by virtue of the one-to-one correspondence between $\vartheta(y)$ and its corresponding invariant measure ([7]), one gets that

$$
\int_{\mathbb{R}_{+}^{2}} h^{\top} y \vartheta(y) d y=\int_{\mathbb{R}_{+}^{2}} h^{\top} y v(d y) .
$$

In view of (2.5), (2.6), (2.7), and (2.8), we can obtain

$$
Y(h)=h^{\top} C^{-1}(B-h) .
$$

Let $\eta=\left(\eta_{1}, \eta_{2}\right)^{\top}$ be the unique solution of the following equation

$$
0=\frac{d Y(h)}{d h}=\frac{d\left(h^{\top}\right)}{d h} C^{-1}(B-h)+\frac{d}{d h}\left[(B-h)^{\top}\left(C^{-1}\right)^{\top}\right] h=C^{-1} B-\left[C^{-1}+\left(C^{-1}\right)^{\top}\right] h .
$$

Therefore, $\eta=\left[C\left(C^{-1}\right)^{\top}+I\right]^{-1} B$. The Hessian matrix is

$$
\frac{d}{d h^{\top}}\left(\frac{d Y(h)}{d h}\right)=\left(\frac{d}{d h}\left(\left(\frac{d Y(h)}{d h}\right)^{\top}\right)\right)^{\top}=\left(\frac{d}{d h}\left(B^{\top}\left(C^{-1}\right)^{\top}-h^{\top}\left[C^{-1}+\left(C^{-1}\right)^{\top}\right]\right)\right)^{\top}=-C^{-1}-\left(C^{-1}\right)^{\top} .
$$

Note that for all $h, C^{-1}+\left(C^{-1}\right)^{\top}$ is positive semi-definite, hence $\eta$ is a global maximum [4]. It then follows from the uniqueness of $\eta$ that if $\left.\Lambda_{2}\right|_{h_{i}=\eta_{i}, i=1,2}>0$ and $\eta_{i} \geqslant 0, i=1,2$, then the optimal harvesting effort is $h^{*}=\eta$ and the maximum of ESY is (2.4).

(ii) First of all, we prove that if $\left.\Lambda_{2}\right|_{h_{i}=\eta_{i}, i=1,2}<0$, or $\eta_{1}<0$, or $\eta_{2}<0$, then the optimal harvesting policy does not exist. Assume that the optimal harvesting effort exists and is denoted as $\widetilde{h}^{*}=\left(\widetilde{h}_{1}^{*}, \widetilde{h}_{2}^{*}\right)^{T}$. Therefore $\widetilde{h}^{*} \in H$. That is to say,

$$
\left.\Lambda_{2}\right|_{h_{i}=\widetilde{h}_{i}^{*}, i=1,2}>0, \quad \widetilde{h}_{i}^{*} \geqslant 0, \quad i=1,2 .
$$

Meanwhile, since $\widetilde{h}^{*}$ is the optimal harvesting effort, then $\widetilde{h}^{*}$ should be the unique solution of Eq. (2.10). Nevertheless, $\eta=\left(\eta_{1}, \eta_{2}\right)^{\top}$ is also the solution of Eq. (2.10). Thereby, $\eta_{i}=\widetilde{h}_{i}^{*} \geqslant 0$ and

$$
\left.\Lambda_{2}\right|_{h_{i}=\eta_{i}, i=1,2}=\left.\Lambda_{2}\right|_{h_{i}=\widetilde{h}_{i}^{*}, i=1,2}>0,
$$

which gives the contradiction. 
Now we are in the position to prove that if $\left.\Lambda_{2}\right|_{h_{i}=\eta_{i}, i=1,2}>0, \eta_{1} \geqslant 0, \eta_{2} \geqslant 0$, and $C^{-1}+\left(C^{-1}\right)^{\top}$ is not positive semi-definite, then the optimal harvesting policy does not exist. Since $\eta_{1} \geqslant 0, \eta_{2} \geqslant 0$ and $\left.\Lambda_{2}\right|_{h_{i}=\eta_{i}, i=1,2}>0$, then $H$ is not an empty set. Consequently, $(2.9)$ holds. Let $\left(\delta_{i j}\right)_{2 \times 2}=C^{-1}+\left(C^{-1}\right)^{\top}$. Then we have

$$
\delta_{11}=2 c_{22} / \Lambda \text {. }
$$

It is easy to see that $\delta_{11}>0$. Consequently, $C^{-1}+\left(C^{-1}\right)^{\top}$ is not negative semi-definite. Hence $C^{-1}+$ $\left(C^{-1}\right)^{T}$ is indefinite. Therefore $Y(h)$ in (2.9) has no extreme points ([4]). This completes the proof.

\section{Examples and numerical simulations}

In this section we will introduce some examples and work out some numerical simulations to illustrate the theoretical results.

First of all, we choose $r_{1}=0.69, r_{2}=0.02, c_{11}=0.39>c_{21}=0.36, c_{22}=0.51>c_{12}=0.09, \sigma_{1}^{2}=\sigma_{2}^{2}=$ $0.1, \mathbb{Y}=[0,+\infty), \lambda(\mathbb{Y})=1, \gamma_{1}(u)=\gamma_{2}(u) \equiv 0.3504, \tau_{1}=10, \tau_{2}=12, \phi_{1}(\theta)=0.3+0.05 \sin \theta, \phi_{2}(\theta)=$ $0.2+0.03 \sin \theta, \theta \in[-12,0]$. Clearly, $C^{-1}+\left(C^{-1}\right)^{\top}$ is positive definite. It then follows from (2.3) that $\eta=\left(\eta_{1}, \eta_{2}\right)^{\top}=(0.2745,0.1173)^{\top}$. It is easy to check that $\eta_{i}>0$ and $\left.\Lambda_{2}\right|_{h_{i}=\eta_{i}, i=1,2}=0.0210>0$. By Theorem 2.6, one can see that

$$
\mathrm{h}_{1}^{*}=\eta_{1}=0.2745, \quad \mathrm{~h}_{2}^{*}=\eta_{2}=0.1173, \quad \mathrm{Y}\left(\mathrm{h}^{*}\right)=\eta^{\top} \mathrm{C}^{-1}(\mathrm{~B}-\eta)=0.2270 .
$$

See Fig. 1, which is the curve of $\mathbb{E}\left[h^{\top} y(t)\right]=\mathbb{E}\left[h_{1} y_{1}(t)+h_{2} y_{2}(t)\right]$. Red line is with $h_{1}=h_{1}^{*}=$ $0.2745, h_{2}=h_{2}^{*}=0.1173$, green line is with $h_{1}=0.4, h_{2}=0.05$, and blue line is with $h_{1}=0.12, h_{2}=0.3$. By Fig. 1, we can see that when $h=h^{*}$ (red line), the expectation of sustainable yield is maximum and $\mathrm{Y}\left(\mathrm{h}^{*}\right)=0.2270$.

Now we choose $r_{1}=0.3, r_{2}=0.02, c_{11}=0.39>c_{21}=0.36, c_{22}=0.51>c_{12}=0.09, \sigma_{1}^{2}=\sigma_{2}^{2}=$ $0.1, \mathbb{Y}=[0,+\infty), \lambda(\mathbb{Y})=1, \gamma_{1}(u)=\gamma_{2}(u) \equiv 0.3504$. Thereby $C^{-1}+\left(C^{-1}\right)^{\top}$ is positive definite. According to $(2.3), \eta=\left(\eta_{1}, \eta_{2}\right)^{\top}=(0.1123,-0.0066)^{\top}$. Clearly, $\eta_{2}<0$. It then follows from (ii) in Theorem 2.6 that the optimal harvesting policy does not exist.

Finally, we choose $r_{1}=0.69, r_{2}=0.2, c_{11}=0.39>c_{21}=0.36, c_{22}=0.51>c_{12}=0.09, \sigma_{1}^{2}=\sigma_{2}^{2}=$ $0.1, \mathbb{Y}=[0,+\infty), \lambda(\mathbb{Y})=1, \gamma_{1}(u)=\gamma_{2}(u) \equiv 0.3504$. By virtue of $(2.3), \eta=\left(\eta_{1}, \eta_{2}\right)^{\top}=(0.3183,0.0121)^{\top}$. Compute that $\left.\Lambda_{2}\right|_{h_{i}=\eta_{i}, i=1,2}=-0.0239<0$. By virtue of (ii) in Theorem 2.6, one can obtain that the optimal harvesting policy does not exist.

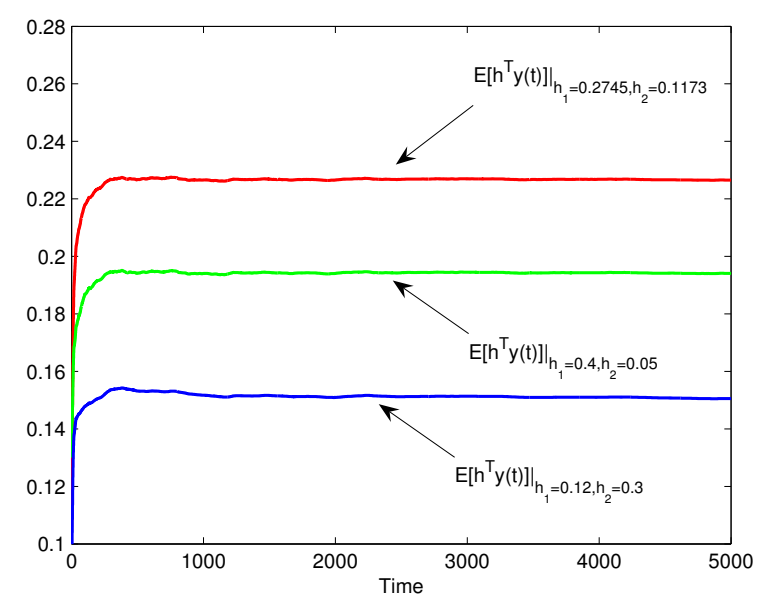

Figure 1: $\mathbb{E}\left[h^{\top} y(t)\right]$ of (1.1) for $r_{1}=0.69, r_{2}=0.02, c_{11}=0.39>c_{21}=0.36, c_{22}=0.51>c_{12}=0.09, \sigma_{1}^{2}=\sigma_{2}^{2}=0.1, \mathbb{Y}=[0,+\infty)$, $\lambda(\mathbb{Y})=1, \gamma_{1}(u)=\gamma_{2}(u) \equiv 0.3504, \tau_{1}=10, \tau_{2}=12, \phi_{1}(\theta)=0.3+0.05 \sin \theta, \phi_{2}(\theta)=0.2+0.03 \sin \theta, \theta \in[-12,0]$. Red line is with $h_{1}=h_{1}^{*}=0.2745, h_{2}=h_{2}^{*}=0.1173$, green line is with $h_{1}=0.4, h_{2}=0.05$ and blue line is with $h_{1}=0.12, h_{2}=0.3$. 


\section{Conclusions}

In this paper, we consider the optimal harvesting problem of a stochastic predator-prey population model with delay and Lévy jumps. Under Assumptions 2.1, $c_{11}>c_{21}$ and $c_{22}>c_{12}$, we establish sufficient and necessary conditions for the existence of an optimal control of system (1.1). Moreover, the explicit forms of the optimal harvesting effort and the maximum of the cost function are obtained. The method used in this paper is the ergodic method, which can avoid solving both the model and the corresponding delay Fokker-Planck equation. The results clearly imply that the optimal harvesting policy has close relationships with white noises and Lévy jumps.

\section{Further research}

Some interesting problems deserve further investigation. In model (1.1), we suppose that species 2 cannot survive in the absence of species 1, i.e., $r_{2}>0$. If this assumption is not true, we can not obtain the critical values between persistence and extinction for $y_{1}$ and $y_{2}$, and hence can not establish the optimal harvesting policy in this case. We leave this problem for further investigation. In addition, one could consider some more realistic but more complex systems, for example, time-varying delays (see, e.g., [23]), regime switching (see, e.g., [9, 20, 31]), fractional models (see, e.g. [27, 28]), or reaction diffusion ([1]). Also it is interesting to study non-automatous models, competitive models, food-chain models or mutualism models $[8,10,17-19,26,29,30]$.

\section{Acknowledgment}

The author thanks Prof. Dumitru Baleanu and the anonymous referees for their careful reading and valuable comments, which greatly improved the presentation of the paper. This study is supported by Natural Science Foundation of Jiangsu Province (No. BK20170067).

\section{References}

[1] C.-Z. Bai, Multiplicity of solutions for a class of non-local elliptic operators systems, Bull. Korean Math. Soc., 54 (2017), 715-729. 5

[2] J.-H. Bao, X.-R. Mao, G. Yin, C.-G. Yuan, Competitive Lotka-Volterra population dynamics with jumps, Nonlinear Anal., 74 (2011), 6601-6616. 1

[3] I. Barbalat, Systems d'equations differentielles d'oscillations nonlinearies, Rev. Roumaine Math. Pures Appl., 4 (1959), 267-270. 2

[4] M. S. Bazaraa, C. M. Shetty, Nonlinear programming, Theory and algorithms, John Wiley \& Sons, New YorkChichester-Brisbane, (1979). 2

[5] J. R. Beddington, R. M. May, Harvesting natural populations in a randomly fluctuating environment, Science, 197 (1977), 463-465. 1, 1

[6] C. W. Clark, Mathematical bioeconomics: the optimal management of renewable resources, Pure and Applied Mathematics, Wiley-Interscience [John Wiley \& Sons], New York-London-Sydney, (1976). 1

[7] G. Da Prato, J. Zabczyk, Ergodicity for infinite-dimensional systems, London Mathematical Society Lecture Note Series, Cambridge University Press, Cambridge, (1996). 2, 2

[8] T. C. Gard, Stability for multispecies population models in random environments, Nonlinear Anal., 10 (1986), 1411-1419. 5

[9] X.-Y. Li, A. Gray, D.-Q. Jiang, X.-R. Mao, Sufficient and necessary conditions of stochastic permanence and extinction for stochastic logistic populations under regime switching, J. Math. Anal. Appl., 376 (2011), 11-28. 5

[10] X.-Y. Li, X.-R. Mao, Population dynamical behavior of non-autonomous Lotka-Volterra competitive system with random perturbation, Discrete Contin. Dyn. Syst., 24 (2009), 523-545. 2, 5

[11] W.-X. Li, K. Wang, Optimal harvesting policy for general stochastic logistic population model, J. Math. Anal. Appl., 368 (2010), 420-428. 1, 1

[12] M. Liu, Optimal harvesting policy of a stochastic predator-prey model with time delay, Appl. Math. Lett., 48 (2015), 102-108. 2

[13] M. Liu, C.-Z. Bai, On a stochastic delayed predator-prey model with Léry jumps, Appl. Math. Comput., 228 (2014), 563-570. 2.2, 2.3 
[14] M. Liu, C.-Z. Bai, Optimal harvesting of a stochastic logistic model with time delay, J. Nonlinear Sci., 25 (2015), $277-289$. 2

[15] M. Liu, C.-Z. Bai, Analysis of a stochastic tri-trophic food-chain model with harvesting, J. Math. Biol., 73 (2016), 597-625. 2

[16] M. Liu, C.-Z. Bai, Dynamics of a stochastic one-prey two-predator model with Lévy jumps, Appl. Math. Comput. 284 (2016), 308-321. 1

[17] M. Liu, C.-Z. Bai, Optimal harvesting of a stochastic delay competitive model, Discrete Contin. Dyn. Syst. Ser. B, 22 (2017), 1493-1508. 5

[18] M. Liu, C.-Z. Bai, Y. Jin, Population dynamical behavior of a two-predator one-prey stochastic model with time delay, Discrete Contin. Dyn. Syst., 37 (2017), 2513-2538.

[19] M. Liu, K. Wang, Q. Wu, Survival analysis of stochastic competitive models in a polluted environment and stochastic competitive exclusion principle, Bull. Math. Biol., 73 (2011), 1969-2012. 5

[20] Q. Luo, X.-R. Mao, Stochastic population dynamics under regime switching, J. Math. Anal. Appl., 334 (2007), 69-84. 5

[21] V. J. Majd, S. Mobayen, An ISM-based CNF tracking controller design for uncertain MIMO linear systems with multiple time-delays and external disturbances, Nonlinear Dynam., 80 (2015), 591-613. 1

[22] S. Mobayen, Design of CNF-based nonlinear integral sliding surface for matched uncertain linear systems with multiple state-delays, Nonlinear Dynam., 77 (2014), 1047-1054.

[23] S. Mobayen, An LMI-based robust tracker for uncertain linear systems with multiple time-varying delays using optimal composite nonlinear feedback technique, Nonlinear Dynam., 80 (2015), 917-927. 5

[24] S. Mobayen, Design of a robust tracker and disturbance attenuator for uncertain systems with time delays, Complexity, 21 (2015), 340-348.

[25] S. Mobayen, F. Tchier, An LMI approach to adaptive robust tracker design for uncertain nonlinear systems with time-delays and input nonlinearities, Nonlinear Dynam., 85 (2016), 1965-1978. 1

[26] H. Qiu, W.-M. Deng, Stationary distribution and global asymptotic stability of a three-species stochastic food-chain system, Turk. J. Math., (2017), accepted. 1, 5

[27] G.-C. Wu, D. Baleanu, Discrete fractional logistic map and its chaos, Nonlinear Dynam., 75 (2014), 283-287. 5

[28] G.-C. Wu, D. Baleanu, H.-P. Xie, F.-L. Chen, Chaos synchronization of fractional chaotic maps based on the stability condition, Phys. A, 460 (2016), 374-383. 5

[29] X.-H. Zhang, D.-Q. Jiang, A. Alsaedi, T. Hayat, Periodic solutions and stationary distribution of mutualism models in random environments, Phys. A, 460 (2016), 270-282. 5

[30] Y. Zhu, M. Liu, Permanence and extinction in a stochastic service-resource mutualism model, Appl. Math. Lett., 69 (2017), 1-7. 5

[31] C. Zhu, G. Yin, On hybrid competitive Lotka-Volterra ecosystems, Nonlinear Anal., 71 (2009), e1370-e1379. 5

[32] X.-L. Zou, W.-X. Li, K. Wang, Ergodic method on optimal harvesting for a stochastic Gompertz-type diffusion process, Appl. Math. Lett., 26 (2013), 170-174. 1, 1

[33] X.-L. Zou, K. Wang, Optimal harvesting for a logistic population dynamics driven by a Lévy process, J. Optim. Theory Appl., 161 (2014), 969-979. 1

[34] X.-L. Zou, K. Wang, Optimal harvesting for a stochastic N-dimensional competitive Lotka-Volterra model with jumps, Discrete Contin. Dyn. Syst. Ser. B, 20 (2015), 683-701. 1 